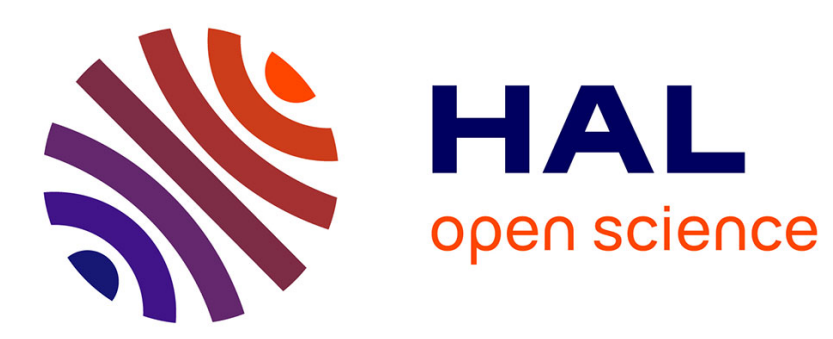

\title{
Recherche de critères de résistance à l'hiver pour le lin
} Yvette Menoux

\section{To cite this version:}

Yvette Menoux. Recherche de critères de résistance à l'hiver pour le lin. Agronomie, 1986, 6 (9), pp.789-796. hal-00884938

\section{HAL Id: hal-00884938 \\ https://hal.science/hal-00884938}

Submitted on 1 Jan 1986

HAL is a multi-disciplinary open access archive for the deposit and dissemination of scientific research documents, whether they are published or not. The documents may come from teaching and research institutions in France or abroad, or from public or private research centers.
L'archive ouverte pluridisciplinaire HAL, est destinée au dépôt et à la diffusion de documents scientifiques de niveau recherche, publiés ou non, émanant des établissements d'enseignement et de recherche français ou étrangers, des laboratoires publics ou privés. 


\title{
Recherche de critères de résistance à l'hiver pour le lin
}

Yvette MENOUX

I.N.R.A., Station de Bioclimatologie, Centre de Recherches de Paris-Grignon, F 78850 Thiverval Grignon

\begin{abstract}
Une double expérimentation en conditions naturelles et en conditions contrôlées est conduite afin de déterminer la validité de certains critères de résistance à l'hiver. La capacité de développer des rameaux secondaires au cours de l'endurcissement et lors de la reprise de végétation déterminée en conditions contrôlées peut être retenue. Complémentairement, la teneur en eau des parties aériennes avant tout traitement thermique est un bon indicateur. L'étude de l'évolution des teneurs en métabolites caractéristiques (sucres totaux solubles, saccharose et proline solubles) ne fournit pas de critères suffisamment discriminants pour le lin.
\end{abstract}

Mots clés additionnels : Capacité de « tallage », état hydrique, sucres solubles, proline, lins d'hiver.

Frost resistance criteria for flax.

\begin{abstract}
Simultaneous experiments were conducted under natural and controlled conditions in order to determine the validity of some frost resistance criteria given in the literature. The ability of plants to develop secondary axes, or "tillering" ability, during hardening and chilling recovery (table 3 and fig. 2a), as well as the water content of aerial parts before temperature treatments (fig. $2 c$ ) could be good indices. These parameters, which are affected by environmental factors, must be determined under standardized conditions. The free proline, free sucrose and free total sugar contents could not be considered for flax, although their changes in value during temperature treatments gave some comparative elements.
\end{abstract}

Additional key words : Tillering ability, water content, soluble sugars, proline, winter flax.

\section{INTRODUCTION}

La recherche de critères de résistance, à une contrainte climatique par exemple, constitue une aide importante à l'amélioration du rendement des plantes par la sélection.

Un programme de relance de la production de graines oléagineuses métropolitaines, de lin en particulier, a été mis en place ces dernières années. Le retour à la pratique de la culture des lins d'hiver a été envisagé. Il est connu en effet que les cultures d'hiver présentent souvent de meilleurs rendements que les cultures de printemps. Nos travaux antérieurs (MENOUX \& FERRON, 1982) ont montré de plus que, pour limiter les risques de verse, autre facteur limitant du rendement, une température fraîche en début de végétation était indispensable ; cette condition se trouve réalisée entre autres à l'automne. Il convient alors que les plantes résistent aux conditions hivernales avec toute la complexité que ces conditions recouvrent.

Ainsi, complémentairement au programme de création de variétés réalisé par nos collègues de la Station
d'Amélioration des Plantes (I.N.R.A.) de Versailles, MM. PLONKA et FOUILLOUX, nous nous sommes attaché à rechercher des critères de résistance à l'hiver permettant un tri facile et précoce de lignées intéressantes.

Ces critères peuvent être de nature morphologique ou physiologique. Les critères morphologiques indiquent une adaptation de la plante soit à éviter un refroidissement trop brusque grâce à l'effet de tampon thermique du sol (réduction d'élongation de la tige entraînant un port en rosette ; DIEHL, 1962), soit à assurer la reprise de végétation au retour des conditions favorables à la croissance : faculté de développer des rameaux secondaires remplaçant les axes détruits par le gel (COUSIN, 1983), système racinaire important (PAQuin \& Pelletier, 1980). Les critères physiologiques traduisent une adaptation à éviter la formation de cristaux de glace intracellulaires provoquant la mort des cellules par destruction mécanique ou par suite de modifications physico-chimiques irréversibles (GENÈVES, 1957 ; TUMANOV \& KRASATSEV, 1959 ; IDLE, 1966 ; Marcellos \& Burke, 1979...). Parmi ceux-ci, 
on peut retenir la faible teneur en eau des tissus (GENEVES, 1955 ; KRASATSEV, 1967 ; TUMANOV, 1967 ; MACKENZIE et al., 1974; DEREUdDRE, 1978 ; WILCOX et al., 1983...) avec comme résultante un ralentissement de croissance (DIEHL, 1962). D'autres critères indiquent le contrôle de la perméabilité cellulaire à l'eau et aux solutés (KACPERSKA-PALACZ et al., 1977 ; LE SAINT, $1977 a$; PALTA et al., 1977 ; BoLDUC et al., 1978) soit par maintien de la pression osmotique, soit par protection des ultrastructures. Le maintien de la pression osmotique est le plus souvent obtenu par la concentration des sucres (SAKAI, 1962; LE SAINT, 1969a; KACPERSKA-PALACZ, 1980 ; Levitt, 1980 ; PaQuin \& Lechasseur, 1982 ; PurVIS \& YELENOSKY, 1982 ; GREEN, 1983...). La protection des ultrastructures est souvent liée à la présence de groupements $\mathrm{SH}^{-}$(LEVITT et al., 1961 ; LEVITT, 1980), à la composition des acides gras polyinsaturés (Grenier et al., 1973 ; Willemot \& Pelletier, 1979 \& 1980 ; MOUTOT et al., 1983 ; WILLEMOT, $1983 \ldots$... La proline jouerait le double rôle de protection des membranes et d'agent osmotique ; elle est considérée aussi comme une forme de réserve de l'azote. Les relations entre forte concentration en proline et résistance aux contraintes climatiques sont abondamment décrites notamment en cas de sécheresse ; parmi les travaux récents relatifs à la résistance au gel, citons ceux de LE SAINT (1969a et $b$ ), LE SAINT $(1977 b)$, LE SAINT \& HUBAC $(1978,1981)$, Hubac \& Vieira Da Silva (1980), Paquin \& SaintPierre (1980), Paquin \& Pelletier (1980), Purvis \& YELENOSKY (1982)...

Il faut noter par ailleurs, que certains facteurs du milieu, tels que teneur en eau du sol, lumière (intensité et photopériode), interviennent sur la résistance au « froid », notamment au cours de l'endurcissement des plantes (PAQUin \& MEHUYs, 1980 ; OUELlet \& DESJARDINS, 1981; RIKIN et al., 1981; ULRICH, 1983). Certains des critères décrits sont donc susceptibles de montrer une variabilité en fonction des conditions de culture ; il en sera tenu compte.

\section{MATÉRIEL ET MÉTHODES}

\section{A. Choix du matériel végétal}

Un travail préliminaire a permis de classer, selon leur pourcentage de survie à l'hiver très rigoureux de 1978-79, les 150 lignées et populations originaires de différents pays ou croisements divers, semées à Versailles par la station d'Amélioration des Plantes. Onze lignées $\left({ }^{*}\right)$ ont été retenues :

- 6 ayant présenté des taux de survie relativement élevés cette année-là (entre 45 et 60 p. 100) : « Hiver 4 », “ Hiver 5 », " Hiver 9 » et « Hiver 1-5», créations I.N.R.A. ; "Plata $2 I$ » et "Lineta $Z$ », importées d'Argentine - tous lins oléagineux.

$\left(^{*}\right)$ Nous donnons au mot lignée, le sens large tel qu'il est défini par R. MARIE (1977) : " toutes les générations successives issues d'un même individu ".
- 3 n'ayant survécu qu'à quelques p. cent : 1 lignée, créée par l'I.N.R.A., issue du croisement "Ariane " $\times$ « Nivale » (lin à fibres $\times$ lin oléagineux) et 2 lins oléagineux : l'un créé par un sélectionneur privé, "Forget 15 », et l'autre originaire d'Italie, "Hiver de Crémone".

- 2 ayant moyennement survécu (20 p. 100) mais intéressantes à d'autres titres : 1 variété de lin oléagineux inscrite en France au catalogue des variétés: "Nivale » et 1 lignée, créée par l'I.N.R.A. issue du croisement «Fany " $\times$ " Nivale » (lin à fibres $\times$ lin oléagineux).

\section{B. Conditions de culture}

Une double expérimentation a été conduite, l'une en conditions normales de la liniculture, l'autre en conditions artificielles répondant au souci de contrôle des facteurs climatiques et à l'étude de leur influence sur l'expression des indicateurs de résistance recherchés.

\section{Conditions normales de la liniculture}

Deux implantations géographiques ont été retenues : Versailles, au climat à tendance océanique, et Colmar, au climat plus contrasté et surtout plus froid.

L'expérimentation s'est poursuivie pendant 5 ans sur les parcelles de l'I.N.R.A. en ces 2 lieux. Les semis en mini-parcelles de $1 \mathrm{~m}^{2}$ par lignée ont été effectués chaque année à la mi-septembre, la densité de semis étant de 1000 graines par $\mathrm{m}$ :.

Sur 2 campagnes (1982-83 et 1983-84), s'est ajouté un essai « influence de la densité de semis» portant sur 4 lignées: "Hiver 5 " et «Hiver 9 »,; "Ariane " $x$ " Nivale" et " Fany" $x$ " Nivale". Le semis est alors effectué à raison de 2000 graines $/ \mathrm{m}^{2}$, densité normale pour le lin à fibres ; soit en tout 14 essais. A chaque essai, 2 répétitions ont été réalisées chaque fois que le nombre de graines et/ou les surfaces de culture disponibles l'ont permis.

Sur ces essais, les critères morphologiques suivants ont été pris en compte : notation du port de la plante avant l'hiver, dénombrement des rameaux secondaires subsistant à la sortie de l'hiver. Le pourcentage de survie a été déterminé. N'ont été suivies que les parcelles n'ayant pas subi d'accidents tels que mauvaise germination des graines, dégâts par les rongeurs..., introduisant une trop forte hétérogénéité dans les cultures avant l'hiver.

\section{Conditions artificielles}

Des conditions édaphiques et nutritives standardisées ont été mises au point pour le lin (MENoux, 1980) : pots de $15 \mathrm{~cm}$ de diamètre, $18 \mathrm{~cm}$ de haut, en matière plastique claire n'absorbant pas le rayonnement pour limiter les effets d'inertie thermique, substrat siliceux de granulométrie moyenne, solution nutritive apportée 2 fois par jour à saturation, 15 plantes par pot (soit une densité de population équivalente à celle obtenue généralement en conditions naturelles).

Les conditions climatiques ont été définies d'après les travaux de LARSEN (1978), LE SAINT \& HUBAC (1978), QUillet (1979) (tabl. 1). 
TABLEAU 1

Séquences climatiques des conditions de culture en enceinte.

Climatic sequences under growth chamber conditions.

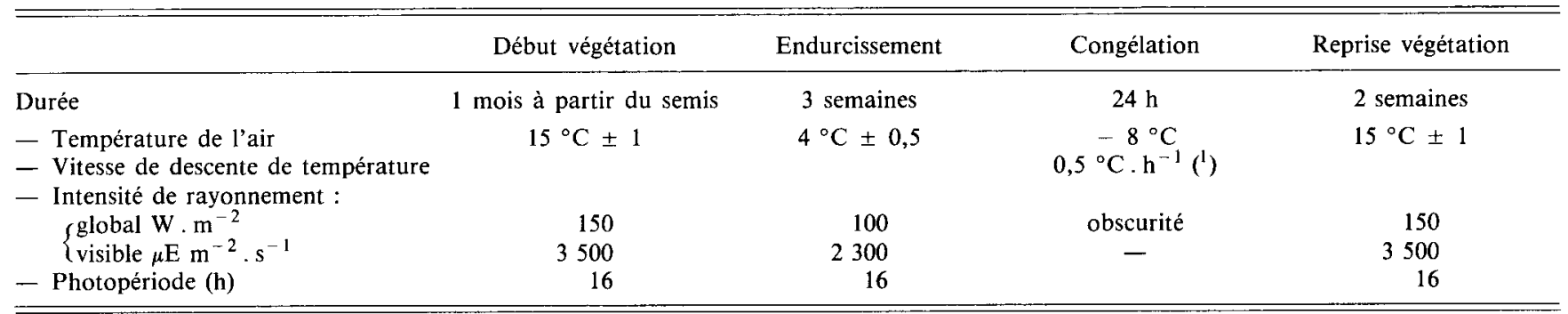

(') Le temps mis à atteindre la température minimale n'est pas inclus dans les $24 \mathrm{~h}$ de congélation.

(l) Time necessary to obtain minimal temperature is not included in freezing time.

Trois lignées seulement ont été étudiées : « Hiver 9 » qui s'est révélée la plus résistante en conditions naturelles, "Forget 15 » et « Hiver de Crémone », les plus sensibles.

Sur 2 séries de culture effectuées sous ces conditions, ont été déterminés, à différents moments du traitement :

- des paramètres morphologiques tels que : somme de toutes les hauteurs des axes secondaires rapportée à la hauteur de l'axe principal et poids de matière sèche des racines et des parties aériennes ;

- des paramètres physiologiques tels que: état hydrique des parties aériennes, teneurs en sucres solubles et acides aminés solubles de différents organes (feuilles jeunes, feuilles adultes, racines). La méthode de dosage des sucres et des acides aminés effectué simultanément à partir d'un même échantillon est celle mise au point par Hubac et al. (1969). Ces analyses sont faites à l'aide d'un autoanalyseur type Jeol.

\section{RÉSULTATS}

\section{A. Critères morphologiques}

1. Relations entre port de la plante, nombre de rameaux secondaires et $p .100$ de survie en conditions naturelles (tabl. 2)

Le " run test " (SIEGEL, 1956), permettant de comparer une distribution ordonnée et un attribut binaire, indique qu'il n'y a pas de relation entre le pourcentage de survie moyen et le port de la plante, d'une part, le nombre de rameaux secondaires, d'autre part. Le port de la plante se révèle le plus constant quelles que soient les conditions climatiques; le nombre de rameaux secondaires montre au contraire une grande variabilité, comme l'indiquent les valeurs élevées du coefficient de variation. Une certaine corrélation entre la valeur du nombre de rameaux secondaires par plante et la densité de population à la reprise de végétation est mise en évidence (fig. 1), illustrant l'influence du rayonnement solaire sur la valeur du critère analysé. Il est à noter que cette influence dif-
TABLEAU 2

Port de la plante, nombre de rameaux secondaires par plante et $p .100$ de survie en conditions naturelles.

Plant habit, number of secondary axes per plant and recovery percentage under natural conditions.

\begin{tabular}{|c|c|c|c|c|}
\hline Lignée & $\begin{array}{c}0 \% \text { survie } \\
\text { moyen } \\
\left.{ }^{1}\right)\end{array}$ & $\begin{array}{c}\text { Port de la } \\
\text { plante } \\
\left({ }^{2}\right)\end{array}$ & $\begin{array}{l}\text { Nbre moyen } \\
\text { de rameaux } \\
\text { secondaires } \\
\text { par plante } \\
\left({ }^{3}\right)\end{array}$ & $\begin{array}{c}\text { C.V. } \\
\left({ }^{4}\right)\end{array}$ \\
\hline Hiver 9 & 95,7 & $\mathrm{R}$ & 1,89 & 20,6 \\
\hline Hiver 4 & 88,7 & $\mathbf{R}$ & 2,22 & 15,8 \\
\hline Hiver 5 & 87,5 & $\mathrm{R}$ & 2,02 & 15,8 \\
\hline Lineta $\mathrm{Z}$ & 84,2 & $\mathrm{E}$ & 2,38 & 19,7 \\
\hline Plata 21 & 80,8 & $\mathrm{E}$ & 1,94 & 23,2 \\
\hline Nivale & 76,5 & $\mathrm{E}$ & 2,24 & 27,7 \\
\hline Hiver 1-5 & 75,3 & $\mathrm{R}$ & 2,13 & 26,3 \\
\hline Fany $\times$ Nivale & 73,8 & $\mathrm{E}$ & 1,58 & 29,0 \\
\hline Ariane $\times$ Nivale & 72,2 & $\mathbf{E}$ & 1,63 & 23,9 \\
\hline Forget 15 & 57,5 & $\mathrm{E}$ & 2,03 & 30,0 \\
\hline Hiver de Crémone & 55,4 & $\mathrm{E}$ & 1,60 & 18,7 \\
\hline
\end{tabular}

(l) et ${ }^{3}$ ) : la valeur moyenne est calculée à partir des valeurs obtenues sur 11 des 14 essais (essais de densité inclus). Les campagnes " Versailles 1979-80 » et «1980-81», « Colmar 1982-83» n'ont pas été prises en compte, toutes les lignées ayant survécu à $100 \mathrm{p}$. 100. La ppds du p. 100 de survie est de 10 environ. Les coefficients de variation C.V. \% $\left({ }^{4}\right)$ s'appliquent à la moyenne du nombre de rameaux secondaires. $\left({ }^{2}\right): \mathrm{E}=$ port érigé $; \mathrm{R}=$ port en « rosette »; ce dernier terme est impropre, en fait l'axe principal se couche à la surface du sol, modifiant ainsi les corrélations entre bourgeon principal et bourgeons cotylédonaires.

$\left({ }^{1}\right)$ and $\left({ }^{3}\right)$ : mean values calculated on the basis of 11 of the 14 experiments (density experiments included). Experiments « Versailles 1979. $80 "$ and " 1980-81", "Colmar 1982-83" are not included, al! lines surviving at $100 \%$. For recovery percentage, $\mathrm{S} E$ is about 10 . Coefficient of variation C.V. $\%\left({ }^{4}\right)$ related to the mean number of secondary axes by plant. $\left({ }^{2}\right): E=$ erect plant $; \mathrm{R}=$ "rosette" habit; in fact, the main axis lies on the soil surface changing hormonal correlations between main bud and cotyledonary ones.

fère largement selon les lignées : elle est très nette pour " Hiver 4 " et «Lineta $Z$ » à fort pourcentage de survie, pour « Forget 15 » à faible pourcentage de survie ; elle est discrète pour "Hiver 9 " à densité 1000 et «Hiver 5 " résistants, pour " Ariane " $\times$ «Nivale " à densité 2000 , «Fany " $\times$ « Nivale » et 

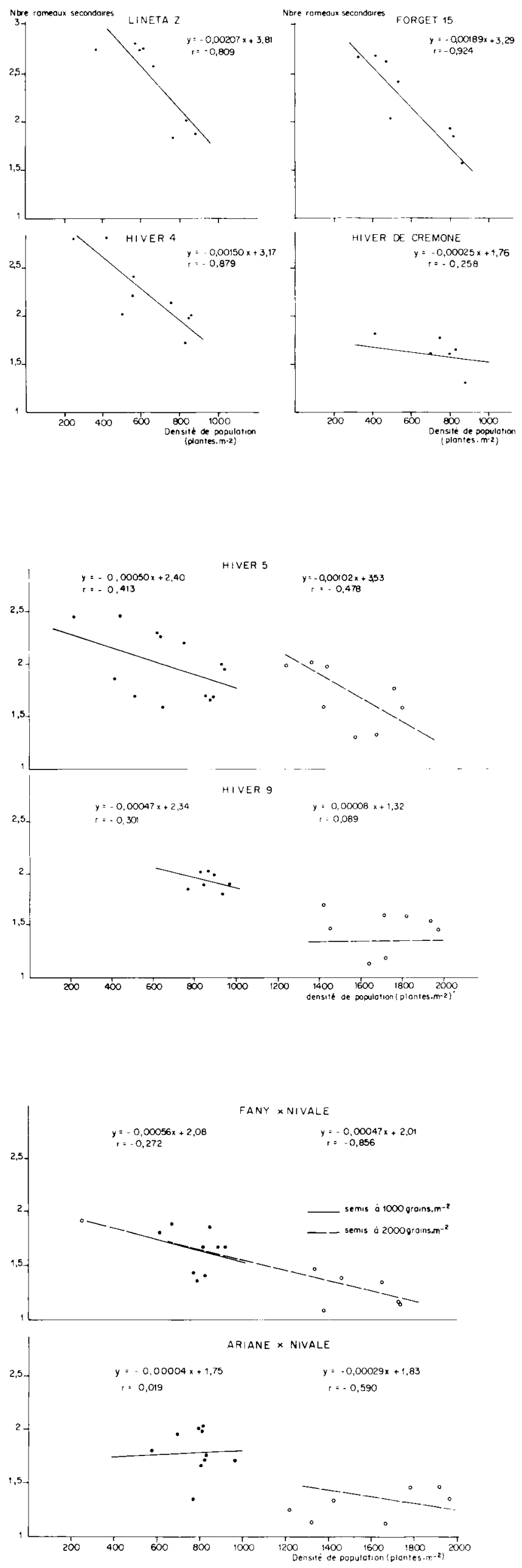

"Hiver de Crémone " sensibles ; elle est nulle pour «Ariane» $\times$ "Nivale» à densité 1000 et "Hiver 9 " à densité 2000 . Aussi, dans une $2^{\mathrm{e}}$ phase de l'analyse, seules les données obtenues sur des populations de densité à la fin de l'hiver comprise entre 800 et 1000 plantes $/ \mathrm{m}^{2}$ sont retenues (tabl. 3, la variabilité est ramenée à un degré habituel pour ce type d'expérimentation). Sur ces nouvelles données, la détermination du coefficient de corrélation de Spearman (SIEGEL, 1956) permet d'isoler un lot homogène de 4 lignées caractérisées par leur résistance à l'hiver et une valeur élevée du critère: "Hiver 9 ", "Hiver 4 », "Hiver 5 » et "Lineta $Z$ ». Une lignée sensible à l'hiver présente la valeur la plus faible de ce critère, "Hiver de Crémone ». Entre ces 2 extrêmes, la variabilité résiduelle ne permet pas un classement strict des autres lignées selon l'hypothèse retenue: "Forget 15 ", avec 1 p. 100 de survie de même valeur qu' " Hiver de Crémone ", apparaît même en contradiction. Une explication est sans doute à rechercher dans le fait que cette lignée montre une forte aptitude à développer des rameaux secondaires en fonction du degré de pénétration du rayonnement solaire dans le

\section{TABLEAU 3}

Nombre de rameaux secondaires par plante pour des populations de même densité. Ordre décroissant des pourcentages de survie à l'hiver comme pour le tableau 2.

Number of secondary axes per plant for the same population density. Recovery percentages listed in decreasing order as in table 2.

\begin{tabular}{lcr}
\hline \multicolumn{1}{c}{ Lignée } & $\begin{array}{c}\text { Nbre moyen de } \\
\text { rameaux } \\
\text { secondaires/plante }\end{array}$ & C.V. \% \\
\hline Hiver 9 & 1,85 & 9,7 \\
Hiver 4 & 1,88 & 6,9 \\
Hiver 5 & 1,85 & 7,6 \\
Lineta Z & 1,94 & 8,2 \\
Plata 21 & 1,70 & 6,9 \\
Nivale & 1,99 & 18,1 \\
Hiver 1-5 & 1,80 & 13,3 \\
Fany $\times$ Nivale & 1,57 & 13,4 \\
Ariane $\times$ Nivale & 1,79 & 11,1 \\
Forget 15 & 1,78 & 10,7 \\
Hiver de Crémone & 1,51 & 10,2 \\
\hline \hline
\end{tabular}

Figure 1

Relations entre le nombre de rameaux secondaires par plante et la densité de population en conditions naturelles.

- « Lineta $Z$ » et « Hiver 4 », p. 100 de survie moyen supérieur à 80 p. 100, "Forget 15 " et " Hiver de Crémone ", p. 100 de survie moyen inférieur à $60 \mathrm{p}$. 100 , semés à 1000 graines $/ \mathrm{m}^{2}$;

- "Hiver 5 » et «Hiver 9 ", lins à graines, p. 100 de survie moyen supérieur à 85 p. 100, "Fany $\times$ Nivale" et « Ariane $\times$ Nivale ", lins mixtes (fibres et graines), p. 100 de survie moven de 70 p. 100 environ, semés à 2 densités : 1000 et 2000 graines $/ \mathrm{m}^{2}$.

Relationship between number of secondary axes per plant and population density under natural conditions. 'Lineta $Z$ ' and 'Hiver 4' had a high survival percentage (higher than $80 \%$ ), 'Forget 15' and 'Hiver de Cremone' a low one (lower than $60 \%$ ), all sou'n at I 000 seeds $/ \mathrm{m}^{2}$; 'Hiver 5' and 'Hiver 9' are linseeds with a mean survival percentage higher than $85 \%$, 'Fany $\times$ Nivale' and 'Ariane $x$ Nivale' mixed flax (fiber and seed) with a mean survival percentage of about $70 \%$, all sown at 2 densities: 1000 and 2000 seeds $/ \mathrm{m}^{2}$. 
couvert. Ce qui conduit à distinguer 2 composantes dans la détermination du degré de résistance à l'hiver à partir du nombre de rameaux secondaires par plante à la sortie de l'hiver: une composante "résistance essentiellement liée au génotype " et une composante « aptitude à récupérer en fonction de facteurs externes $\gg$.

\section{Développement des rameaux secondaires en condi- tions artificielles (fig. 2a)}

Le rapport : somme de toutes les hauteurs des axes secondaires/hauteur de l'axe principal présente des valeurs significativement plus faibles chez les 2 lignées sensibles que chez la résistante.
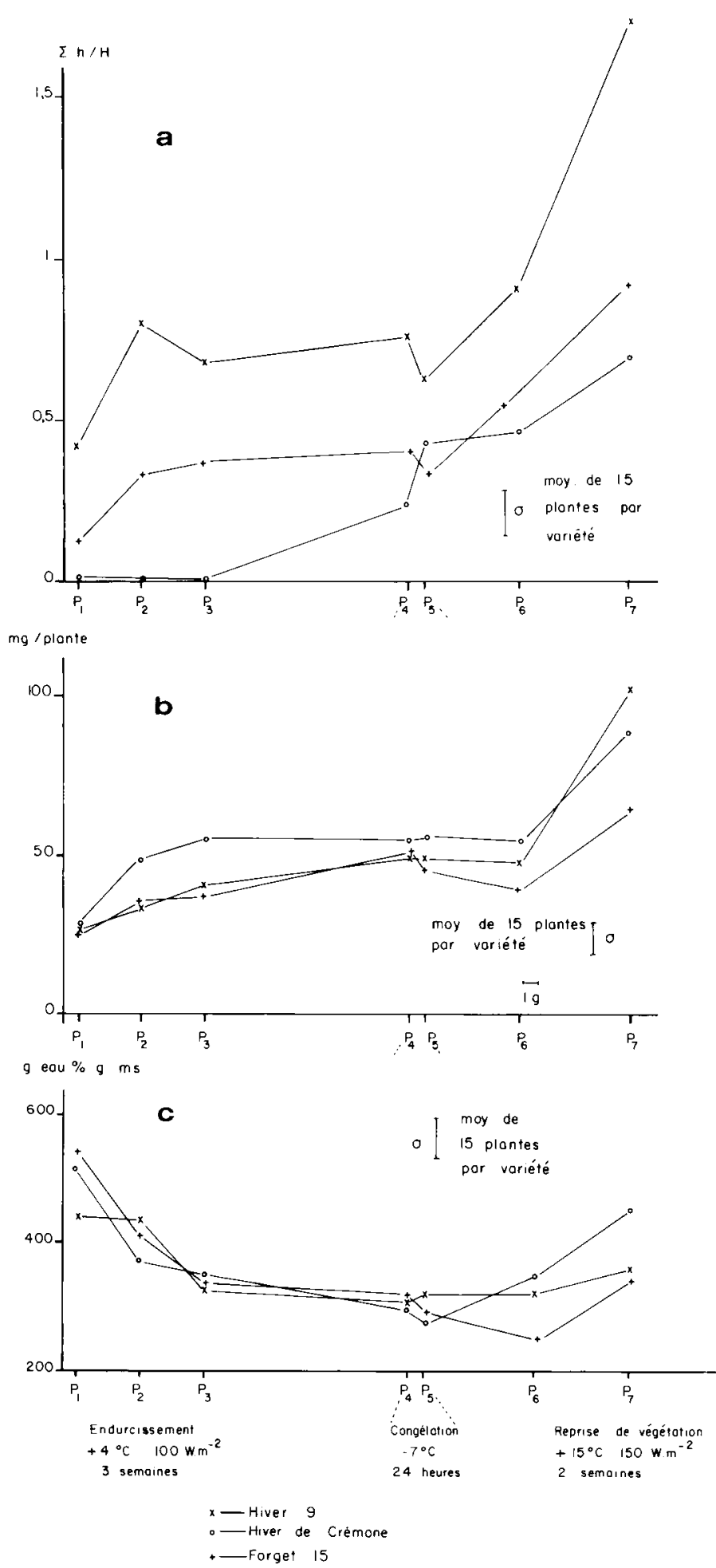

\section{Importance du système racinaire (fig. 2b)}

Il n'apparaît pas de différence significative du poids de matière sèche des racines entre les 3 lignées.

\section{B. Critères physiologiques}

\section{Etat hydrique des parties aériennes (fig. 2c)}

Avant endurcissement (P1), les tissus des parties aériennes des lignées sensibles sont plus hydratés que ceux de la lignée résistante. La différence de l'ordre de 20 p. 100 est significative. Elle disparaît ensuite, entraînant ainsi une perte en eau plus importante chez les 2 lignées sensibles. Deux semaines après la reprise de végétation, seule «Hiver de Crémone » a de nouveau une hydratation supérieure aux 2 autres.

\section{Concentration en sucres solubles totaux (tabl. 4)}

Les résultats sont ici rapportés à la masse d'eau des tissus dans la mesure où il s'agit d'un critère osmotique. Les feuilles jeunes de la lignée résistante ont une concentration en sucres solubles totaux qui augmente fortement lors de l'endurcissement. Cette concentration élevée se maintient ensuite. Chez les lignées sensibles, la concentration reste stable, voire diminue légèrement jusqu'à la fin de la congélation ; elle diminue fortement lors de la reprise de végétation. Ainsi, les feuilles jeunes se comportent selon l'hypothèse de départ : une pression osmotique élevée peut assurer une protection plus efficace contre la formation de cristaux de glace intracellulaires. Ce type de comportement reste valable pour les racines, mais les différences sont moins accentuées entre les 3 lignées.

\section{Critères métaboliques (tabl. 5)}

L'étude des critères les plus caractéristiques de la résistance: saccharose, produit d'accumulation du métabolisme carboné, et proline, forme de réserve de l'azote et agent protecteur, fait apparaître 2 faits con-

Figure 2

Evolution de certains paramètres en conditions contrôlées au cours des différentes séquences thermiques - moyenne de 15 plantes, Ire série - «Hiver 9 ": résistant; "Hiver de Crémone " et « Forget $15 "$ : sensibles.

a-évolution du rapport: somme de toutes les hauteurs des axes secondaires sur hauteur de l'axe principal: $h / H$;

$b$ - évolution de la masse de matière sèche des racines;

$c$ - évolution de la teneur en eau des parties aériennes.

Sur l'axe des abscisses, sont indiqués les moments des prélèvements: $P_{1}=$ avant endurcissement: $P_{2}, P_{3}, P_{4}=$ après respectivement $4,8,21 j$ d'endurcissement; $P_{5}=$ après $I j$ de congélation ; $P_{6}$ et $P_{7}=$ après respectivernent 6 et $14 j$ de reprise de végétation.

Changes in some parameters during different temperature sequences under controlled conditions - mean of 15 plants; Ist serie 'Hiver 9' - resistant line; 'Hiver de Cremone' and 'Forget 15' : sensitive lines.

$a-$ changes in the ratio: sum of height all secondary axes/main axis height: $h / H$;

$b$ - changes in root matter weight;

$c$ - changes in water content of aerial parts.

On $x$-axis times of sampling: $P_{1}=$ before hardening; $P_{2}, P_{3}$, $P_{4}=$ after respectively $4,8,21$ days of hardening; $P_{5}=$ after $I$ day of freezing ; $P_{6}$ and $P_{7}=$ after respectively 6 and 14 days of recovery. 
TABLEAU 4

Evolution de la concentration en sucres totaux solubles dans différentes parties de la plante.

Les valeurs sont exprimées en $\mu$ moles par $m g$ d'eau $: t_{i}=$ avant endurcissement $; t_{1}=$ après endurcissement $; t_{2}=$ après congélation ;

$t_{3}=$ après 2 semaines de reprise de végétation ; $H 9=$ Hiver 9, résistant; $H C=$ Hiver de Crémone et $F 15=$ forget 15 , sensibles.

Changes in total soluble sugar concentrations in different plant organs. Values expressed as $\mu$ mole per mg water. $t_{i}=$ before hardening $; t_{1}=$ after hardening $; t_{2}=$ after freezing $; t_{3}=$ after 2 wk-recovery.

\begin{tabular}{|c|c|c|c|c|c|c|c|c|}
\hline & & $t_{i}$ & $t_{1}$ & $t_{2}$ & $t_{3}$ & $t_{1} / t_{i}$ & $\mathrm{t}_{2} / \mathrm{t}_{\mathrm{i}}$ & $\mathrm{t}_{3} / \mathrm{t}_{\mathrm{i}}$ \\
\hline $\begin{array}{l}\text { Feuilles } \\
\text { jeunes }\end{array}$ & $\left\{\begin{array}{l}\mathrm{H} 9 \\
\mathrm{HC} \\
\mathrm{F} 15\end{array}\right.$ & $\begin{array}{l}25,6 \\
36,0 \\
48,9\end{array}$ & $\begin{array}{l}84,5 \\
25,2 \\
39,1\end{array}$ & $\begin{array}{l}87,0 \\
28,8 \\
88,0\end{array}$ & $\begin{array}{r}64,0 \\
7,2 \\
19,6\end{array}$ & $\begin{array}{l}3,3 \\
0,7 \\
0,8\end{array}$ & $\begin{array}{l}3,4 \\
0,8 \\
1,8\end{array}$ & $\begin{array}{l}2,5 \\
0,2 \\
0,4\end{array}$ \\
\hline $\begin{array}{l}\text { Feuilles } \\
\text { adultes }\end{array}$ & $\left\{\begin{array}{l}\mathrm{H} 9 \\
\mathrm{HC} \\
\mathrm{F} 15\end{array}\right.$ & $\begin{array}{r}82,0 \\
102,6 \\
163,0\end{array}$ & $\begin{array}{l}246,0 \\
369,4 \\
570,5\end{array}$ & $\begin{array}{l}155,8 \\
338,6 \\
456,4\end{array}$ & & $\begin{array}{l}3,0 \\
3,6 \\
3,5\end{array}$ & $\begin{array}{l}1,9 \\
3,3 \\
2,8\end{array}$ & $\begin{array}{l}- \\
-\end{array}$ \\
\hline Racines & $\left\{\begin{array}{l}\mathrm{Ha}^{19} \\
\mathrm{HC} \\
115\end{array}\right.$ & $\begin{array}{r}86 \\
141 \\
131\end{array}$ & $\begin{array}{l}498,8 \\
352,5 \\
484,7\end{array}$ & $\begin{array}{l}301,0 \\
282,0 \\
550,2\end{array}$ & $\begin{array}{l}111,8 \\
141,0 \\
104,8\end{array}$ & $\begin{array}{l}5,8 \\
2,5 \\
3,7\end{array}$ & $\begin{array}{l}3,5 \\
2,0 \\
4,2\end{array}$ & $\begin{array}{l}1,3 \\
1,0 \\
0,8\end{array}$ \\
\hline
\end{tabular}

TABLEAU 5

Evolution des teneurs en saccharose et en proline.

Les valeurs sont exprimées en pmoles par mg de matière sèche, même symboles que pour le tableau 4.

Changes in sucrose and proline levels.

values expressed as pmole per mg dry matter weight. Same symbols as in table 4.

\begin{tabular}{|c|c|c|c|c|c|c|c|c|}
\hline & & $t_{i}$ & $t_{1}$ & $t_{2}$ & $\mathrm{t}_{3}$ & $t_{1} / t_{i}$ & $\mathrm{t}_{2} / \mathrm{t}_{\mathrm{i}}$ & $\mathrm{t}_{3} / \mathrm{t}_{\mathrm{i}}$ \\
\hline \multicolumn{9}{|c|}{ Saccharose } \\
\hline $\begin{array}{l}\text { Feuilles } \\
\text { jeunes }\end{array}$ & $\left\{\begin{array}{l}\mathrm{H} 9 \\
\mathrm{HC} \\
\mathrm{F} 15\end{array}\right.$ & $\begin{array}{l}15,2 \\
17,6 \\
38,6\end{array}$ & $\begin{array}{l}56,3 \\
17,6 \\
30,9\end{array}$ & $\begin{array}{r}30,4 \\
56,3 \\
177,6\end{array}$ & $\begin{array}{r}107,9 \\
12,3 \\
19,3\end{array}$ & $\begin{array}{l}3,7 \\
1 \\
0,8\end{array}$ & $\begin{array}{l}2,0 \\
3,2 \\
4,6\end{array}$ & $\begin{array}{l}7,1 \\
0,7 \\
0,5\end{array}$ \\
\hline $\begin{array}{l}\text { Feuilles } \\
\text { adultes }\end{array}$ & $\left\{\begin{array}{l}\mathrm{H} 9 \\
\mathrm{HC} \\
\mathrm{F} 15\end{array}\right.$ & $\begin{array}{l}37,4 \\
45,9 \\
13,3\end{array}$ & $\begin{array}{l}41,1 \\
36,7 \\
16,0\end{array}$ & $\begin{array}{c}0 \\
0 \\
22,6\end{array}$ & & $\begin{array}{l}1,1 \\
0,8 \\
1,2\end{array}$ & $\begin{array}{l}0 \\
0 \\
1,7\end{array}$ & $\begin{array}{l}- \\
-\end{array}$ \\
\hline Racines & $\left\{\begin{array}{l}\mathrm{H} 9 \\
\mathrm{HC} \\
\mathrm{F} 15\end{array}\right.$ & $\begin{array}{r}3,1 \\
11,7 \\
7,7\end{array}$ & $\begin{array}{l}31,0 \\
48,0 \\
29,3\end{array}$ & $\begin{array}{l}23,9 \\
26,9 \\
41,6\end{array}$ & $\begin{array}{l}31,06 \\
80,7 \\
11,5\end{array}$ & $\begin{array}{l}10 \\
4,1 \\
3,8\end{array}$ & $\begin{array}{l}7,7 \\
2,3 \\
5,4\end{array}$ & $\begin{array}{l}10 \\
6,9 \\
1,5\end{array}$ \\
\hline \multicolumn{9}{|l|}{ Proline } \\
\hline $\begin{array}{l}\text { Feuilles } \\
\text { jeunes }\end{array}$ & $\left\{\begin{array}{l}\mathrm{H} 9 \\
\mathrm{HC} \\
\mathrm{F} 15\end{array}\right.$ & $\begin{array}{r}18,5 \\
4,5 \\
11,9\end{array}$ & $\begin{array}{r}107,3 \\
23,8 \\
183,3\end{array}$ & $\begin{array}{r}223,8 \\
8,1 \\
152,3\end{array}$ & $\begin{array}{l}14,8 \\
10,8 \\
16,7\end{array}$ & $\begin{array}{r}5,8 \\
5,3 \\
15,4\end{array}$ & $\begin{array}{r}12,1 \\
1,8 \\
12,8\end{array}$ & $\begin{array}{l}0,8 \\
2,4 \\
1,4\end{array}$ \\
\hline $\begin{array}{l}\text { Feuilles } \\
\text { adultes }\end{array}$ & $\left\{\begin{array}{l}\mathrm{H} 9 \\
\mathrm{HC} \\
\mathrm{F} 15\end{array}\right.$ & $\begin{array}{r}6,6 \\
40,3 \\
34,5\end{array}$ & $\begin{array}{r}71,3 \\
129,0 \\
106,9\end{array}$ & $\begin{array}{l}132,0 \\
169,3 \\
131,1\end{array}$ & & $\begin{array}{r}10,8 \\
3,2 \\
3,1\end{array}$ & $\begin{array}{l}20 \\
4,2 \\
3,8\end{array}$ & $\begin{array}{l}- \\
- \\
-\end{array}$ \\
\hline Racines & $\left\{\begin{array}{l}\mathrm{H} 9 \\
\mathrm{HC} \\
\mathrm{F} 15\end{array}\right.$ & $\begin{array}{l}2,0 \\
4,0 \\
3,2\end{array}$ & $\begin{array}{l}24,2 \\
24,4 \\
41,9\end{array}$ & $\begin{array}{l}36,0 \\
11,2 \\
12,2\end{array}$ & $\begin{array}{l}2,0 \\
4,0 \\
3,2\end{array}$ & $\begin{array}{r}12,1 \\
6,1 \\
13,1\end{array}$ & $\begin{array}{r}13,0 \\
2,8 \\
3,8\end{array}$ & $\begin{array}{l}1 \\
1 \\
1\end{array}$ \\
\hline
\end{tabular}

tradictoires : les teneurs initiales (ti) en ces métabolites sont dans l'ensemble, dans les conditions expérimentales réalisées, plus élevées chez les 2 lignées sensibles que chez la résistante, contrairement à ce qui est trouvé généralement pour d'autres espèces végétales. Au cours des diffférentes séquences thermiques, l'évolution des teneurs rapportées aux teneurs initiales est, par contre, conforme au comportement attendu chez "Hiver 9" et "Hiver de Crémone": plus forte accumulation relative de ces produits chez la lignée résistante que chez la sensible. "Forget 15 " ne suit pas cette règle. Les mêmes types de réponse ont été obtenus pour les 2 séries de culture réalisées.
L'analyse des autres métabolites : sucres réducteurs et autres acides aminés n'a pas fourni d'éléments de comparaison entre les 2 catégories de plantes étudiées.

\section{DISCUSSION ET CONCLUSIONS}

De l'ensemble des résultats obtenus, il ressort que seul le paramètre décrivant un potentiel de remplacement des axes détruits puisse être retenu. Il a une origine génétique ; il se détermine facilement en dénom- 
brant les rameaux secondaires présents à la reprise de végétation. Son expres̉sion pouvant ètre fortement liée à l'environnement, il est recommandé de pratiquer les essais en conditions contrôlées. L'état hydrique des parties aériennes déterminé dans ces mêmes conditions peut aussi se révéler un bon indicateur ; les différences obtenues sont toutefois moins significatives.

Les critères métaboliques étudiés ne permettent pas chez le lin une séparation sûre entre plantes résistantes et plantes sensibles à l'hiver si l'on ne prend en compte que les valeurs absolues des concentrations en sucres solubles totaux (indicateur de la pression osmotique des tissus) ou celles des taux de saccharose et de proline (indicateurs de résistance des métabolismes respectivement carboné et azoté). Toutefois, si l'on compare la lignée résistante "Hiver 9 " à la lignée sensible "Hiver de Crémone", l'évolution de ces indicateurs (valeurs obtenues au cours des différentes séquences thermiques rapportées à la valeur initiale) apparaît discriminante pour les feuilles jeunes et à un degré moindre pour les racines, c'est-à-dire pour des organes au rôle déterminant de la survie de la plante. Pour cet ensemble de critères métaboliques, la lignée sensible " Forget 15 » ne montre pas toujours les différences attendues. Ce fait est peut-être à relier à sa productivité relativement élevée: la production de matière sèche en conditions contrôlées est environ 1,5 fois supérieure à celle de "Hiver 9 " et " Hiver de Crémone » $(260 \mathrm{mg}$ pour "Forget 15 », 170 pour "Hiver 9 » et "Hiver de Crémone", moyenne de 30 plantes, les 2 séries confondues). Rappelons que les conditions de culture ont été établies afin de ne pas induire de dégâts irréversibles. En conditions naturelles, la production de graines est dans le même rapport si l'on ne considère que les essais où «Forget 15 » a survécu à plus de 80 p. $100: 440 \mathrm{~g} . \mathrm{m}^{2}$ contre $300 \mathrm{~g} \cdot \mathrm{m}^{2}$ pour "Hiver 9 " et "Hiver de Crémone". Cette différence disparaît lorsque la moyenne est calculée sur l'ensemble des essais; ainsi l'aptitude que montre «Forget 15 » à développer des rameaux secondaires quand la densité de population diminue, donc après une forte atteinte de l'hiver, ne permet pas une compensation satisfaisante quant au rendement en graines. Il apparaît donc que, dans les conditions expérimentales réalisées, il soit difficile d'avoir une lignée résistante à l'hiver et forte productrice de graines.

Pour la fibre où l'on recherche des plantes monotiges, les observations faites dans cet article nous rendent pessimiste quant à la possibilité de sélection de lin d'hiver à fibres.

Reçu le 19 avril 1985. Accepté le 14 mai 1986.

\section{REMERCIEMENTS}

Nous tenons à exprimer nos remerciements à la Direction de l'Institut technique du Lin pour son concours fïnancier, Mmes Hubac et LE SAINT pour leurs conseils précieux, la Direction du Domaine expérimental de l'I.N.R.A.-Colmar, le service de Mme Hubac au Phytotron du C.N.R.S. de Gif-sur-Yvette et la Station d'Amélioration des plantes (I.N.R.A.) de Versailles pour leur aide technique.

\section{RÉFÉRENCES BIBLIOGRAPHIQUES}

Bolduc R., Rancourt L., Dolbec P., Chouinard-Lavoie L., 1978. Mesure de l'endurcissement au froid et de la viabilité des plantes exposées au gel par le dosage des phosphatases acides libres. Can. J. Plant Sci., 58, 1007-1018.

Cousin R., 1983. Résistance au froid et sélection chez le pois. Sel. fr., 31, 17-28.

Dereuddre J., 1978. Effet de divers types de refroidissements sur la teneur en eau et sur la résistance au gel des bourgeons de rameaux d'épicea en vie ralentie. Physiol. vég., 16 (3), 469-489.

Diehl R., 1962. Quelques aspects de la résistance au froid chez les céréales d'hiver. Ann. Amélior. Plantes, 2, 257-303.

Genèves L., 1955. Recherches sur les effets cytologiques du froid. Rev. Cytol. Biol. vég., 16, 1-178.

Genèves L., 1957. Influence du froid hivernal puis du réchauffement sur les feuilles d'Iris germanica L. Rev. gén. Bot., 64, 1-30.

Green D. G., 1983. Soluble sugar changes occuring during cold hardening of spring wheat, fall rye and alfalfa. Can. J. Plant Sci., 63, 415-420.

Grenier G., Mazliak P., Trémolières A., Willemot C., 1973. Influence du froid sur la synthèse des acides gras dans les racines de deux variétés de luzerne, l'une très résistante et l'autre moins résistante au froid. Physiol. vég., 11, 253-265.

Hubac C., Guerrier D., Ferran J., 1969. Résistance à la sécheresse du Carex pachystylis (J. Gay) plante du désert du Négev. Oecol. Plant, IV, 325-346.

Hubac C., Vieira Da Silva J., 1980. Indicateurs métaboliques de contraintes mésologiques. Physiol. vég., 18 (1), 45-53.

Idle D. B., 1966. The photography of ice formation in plant tissue. Ann. Bot., 30, 199-207.

Kacperska-Palacz A., 1980. Metabolic consequences of temperature stress $=$ injury or adaptation? Fed. Eur. Soc. Plant Physiol. Il. Congress, Santiago de Compostella, Abs., 58.

Kacperska-Palacz A., Dlugokecka E., Breitenwald J., Wcislinska B., 1977. Physiological mechanisms of frost tolerance. Subcellular localization and some physical-chemical properties of protein fractions accumulated under cold treatment. Biol. Plant, 19, 18-26.

Krasavtsev O. A., 1967. Frost hardening of woody plants at temperatures below zero. In: Asahina E. : "Cellular injury and resistance in freezing organisms ». Inst. low temperature Sci. Hokkaido Univ., Japon, 131-141.

Larsen A., 1978. Freezing tolerance in grasses. Methods for testing in controlled environments. Med. Norg. Landbr. Hogsk., 57 (23), $1-56$.

Le Saint A. M., 1969a. Variations comparées des teneurs en proline libre et en glucides solubles, en relation avec l'inégale sensibilité au gel des organes de plantes de chou de Milan cult. Pontoise. C.R. Acad. Sci. Paris, série D, 268, 310-313.

Le Saint-Quervel A. M., 1969b. Corrélations entre la résistance au gel, l'éclairement et la teneur en proline libre chez le chou de Milan cult. Pontoise. C.R. Acad. Sci. Paris, série D, 269, 1423-1426.

Le Saint-Quervel A. M., 1977a. Recherche et utilisation d'une méthode d'évaluation de la résistance au gel de fragments de feuilles. C.R. Acad. Sci. Paris, série D, 284, 41-44.

Le Saint A. M., 1977b. Maintien et perte de la résistance au gel chez des fragments de limbes foliaires prélevés sur du chou de Milan cult. Pontoise endurci naturellement. C.R. Acad. Sci. Paris, série D, 285, 1435-1437.

Le Saint A. M., Hubac C., 1978. Endurcissement au gel de fragments de feuilles de chou de Milan cult. "Pontoise " maintenus en survie. Possibilités de l'endurcissement et variations concomitantes de la proline libre. C.R. Acad. Sci. Paris, série D, 286, 1347-1349. 
Le Saint A. M. Hubac C., 1981. Analyse du comportement de feuilles in situ et de fragments isolés de limbe foliaire lors d'un endurcissement au gel du chou de Milan cult. Pontoise. 106 Congr. Natl. Soc. Savantes, Perpignan, Sciences, Fasc. II, 89 96.

Levitt J., 1980. Responses of plants to environmental stresses, vol. 1 Kozlowski T. T., $2^{\mathrm{e}}$ éd., Acad. Press N.Y., 497 p.

Levitt J., Sullivan C. Y., Johansson N. O., Pettit R. M., 1961 Sulfhydryls. A new factor in frost resistance. I. Changes in SH content during trost hardening. Plant Physiol., 36, 611-616.

Mac Kenzie J. S., Weiser C. J., Lip H., 1974. Changes in water relations of Cornus stolonifera during cold acclimation. J. Am. Soc. Hortic. Sci., 99, 223-226.

Marcellos H., Burke M. J., 1979. Frost injury in wheat. Ice formation and injury in leaves. Aust. J. Plant Physiol., 6, 513-521.

Marie R., 1977. Glossaire de l'amélioration génetique du riz. Suppl.

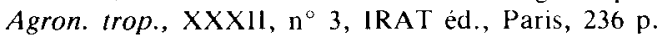

Menoux Y., 1980. Effets et post-effets d'une sécheresse édaphique temporaire modérée sur la croissance du lin : influence des conditions énergétiques du milieu. Acta Oecol., Oecol. Plant, 1, 55-69.

Menoux Y., Ferron F., 1982. Effets d'une déshydratation sur l'ouverture stomatique et le métabolisme carboné à la lumière des feuilles de lin (Linum usitassimum L. cv. "Hera »). Photosvnthelica, 16 (4), 526-532.

Menoux Y., Katz E., Eyssautier A., Parcevaux S. de, 1982. Résistance à la verse du lin textile. Influence du milieu et critères de sélection proposés. Agronomie, 2 (2), 173-180.

Moutot F., Justin A. M., Mazliak P., Jolivet E., 1983. Effets de la température sur la désaturation des acides gras foliaires de lins textiles et oléagineux. Physiol. vég., 21 (1), 29-38.

Ouellet C. E., Desjardins R. L., 1981. Interprétation des relations entre le climat et la survie à l'hiver de la luzerne par l'analyse des corrélations. Can. J. Plant Sci., 61 (4), 945-954.

Palta J. P., Levitt J., Stadelmann E. J., 1977. Freezing injury in onion bulb cells. I. Evaluation of the conductivity method and analysis of ion and sugar efflux from injured cells. Plant Physiol. $60,396-397$

Paquin R., Lechasseur P., 1982. Acclimatation naturelle de la luzerne (Medicago media Pers.) au froid. II. Variations de la teneur en sucres totaux des feuilles et des collets. Acta Oecol., Oecol. Plant, 3 (1), 27-38.

Paquin R., Mehuys G., 1980. Influence of soil moisture on cold tolerance of alfalfa. Can. J. Plant Sci., 60, 139-147.
Paquin R., Pelletier H., 1980. Influence de l'environnement sur l'acclimatation au froid de la luzerne (Medicago media Pers.) et sa résistance au gel. Can. J. Plant Sci., 60, 1351-1366.

Paquin R., Saint Pierre J. C., 1980. Endurcissement, résistance au gel et contenu en proline libre de la fléole des prés (Phleum pratense L.). Can. J. Plant Sci., 60, 525-532.

Purvis A. C., Yelenosky G., 1982. Sugar and proline accumulation in grape-fruit flavedo and leaves during cold hardening of young trees. J. Am. Soc. Hortic. Sci., 107 (2), 222-226.

Quillet A., 1979. Contribution à la mise au point d'un test artificiel déterminant la résistance du lin (Linum usitatissimum $L$.) au froid. Mémoire de fin d'études, I.S.A. Beauvais, $69 \mathrm{p}$.

Rikin A., Gitler C., Atsmon D., 1981. Chilling injury in cotton (Gossypium hirsutum L.) : light requirement for the reduction of injury and for the protective effects of abscissic acid. Plant Cell Physiol., 22 (3), 453-460.

Sakai A., 1962. Mechanism of the protective action of sugars against frost injury in plant cells. Nature, 193 (4810), 89-90.

Siegel S., 1956. Non parametric statistics for the behavioral sciences. In Series in psychology, Mac Graw Hill éd., New York, 312 p.

Tumanov I. I., 1967. The frost hardening process in plants. In Troshin A. S. : "The cell and environmental temperature », éd., Pergamon Press, 6-14.

Tumanov I. I., Krasavtsev O. A., 1959. Hardening of northern woody plants by temperatures below zero. Sov. Plant Physiol., 6 (6), 654-667.

Ulrich R., 1983. Les problèmes physiologiques posés par la résistance au gel. Sel. fr., 31, 5-9.

Wilcox D. A., Davies F. S., Buchanan D. W., 1983. Root temperature, water relations and cold hardiness in two citrus rootstocks. $J$ Am. Soc. hortic. Sci., 108 (2), 318-321.

Willemot C., 1983. Rapid degradation of polar lipids in frost damaged winter wheat crown and root tissue. Phytochemistry, 22 (4), $861-863$.

Willemot C., Pelletier L., 1979. Effect of drought on frost resistance and fatty acid content of young winter wheat plants. Can. J. Plant Sci., 59 (3), 639-643.

Willemot C., Pelletier L., 1980. Influence de la lumière et de la température sur la teneur en acide linolénique et la résistance au gel du blé d'hiver. Can. J. Plant Sci., 60, 349-355. 